\title{
Nivel de Ecoeficiencia en la Cooperativa Agraria Cafetalera Divisoria LTDA y la Cooperativa Agraria Industrial Naranjillo
}

\section{Eco-efficiency level in Cooperativa Agraria Cafetalera Divisoria LTDA and Cooperativa Agraria Industrial Naranjillo}

Reiner Pedro Gabriel Reátegui Inga ${ }^{1 *}\left(\mathbb{D}\right.$, Manuel Emilio Reátegui Inga ${ }^{1} \mathbb{D}$, Fátima de la Merced Pinglo Jurado ${ }^{1}$, Alex Lenin Guivin Guadalupe ${ }^{1}$ (D)

\section{RESUMEN}

El presente trabajo de investigación busca evaluar el nivel de ecoeficiencia en las instalaciones de la Cooperativa Agraria Cafetalera Divisoria LTDA (CACD) y las instalaciones de la Cooperativa Agraria Industrial Naranjillo (COOPAIN). Para ello se utilizó la Guía de ecoeficiencia para empresas del Ministerio de Ambiente de Perú y el Procedimiento de identificación de aspectos y valoración de impactos ambientales de la Universidad del Atlántico. Se identificó los impactos ambientales y su valoración, como complemento a la elaboración del ecobalance para medir la ecoeficiencia en las cooperativas. Para determinar el ecobalance se evaluó el consumo de agua, el consumo de energía eléctrica, el consumo de materiales, la gestión de residuos y las emisiones de CO2. En COOPAIN, se identificaron tres tipos de contaminación, la generación de residuos no peligrosos con un impacto de bajo a medio, las emisiones atmosféricas y contaminación por ruidos con niveles de impacto medios, y la existencia de consumo de energía y agua con un impacto medio. En cuanto al ecobalance obtuvo mayor consumo en agua y energía eléctrica. Por otro lado, en CACD, solamente se identificó un solo tipo de contaminación, la generación de residuos no peligrosos y la existencia de consumo de energía, ambos en un nivel de impacto bajo. En lo referente al ecobalance, obtuvo mayor consumo de materiales, mayor gestión de residuos y mayores emisiones de CO2. Por último, el índice de ecoeficiencia indico que CACD fue relativamente más ecoeficiente que COOPAIN.

Palabras claves: ecoeficiencia; consumo de agua; consumo de energía; consumo de materiales; gestión de residuos; emisión de $\mathrm{CO} 2$.

\begin{abstract}
The present work of investigation seeks to evaluate the level of ecoefficiency in the facilities of the Agrarian Coffee Cooperative Divisoria LTDA (CACD) and the facilities of the Agrarian Industrial Cooperative Naranjillo (COOPAIN). To this end, the Eco-Efficiency Guide for Companies of the Peruvian Ministry of Environment and the Procedure for the Identification of Aspects and Assessment of Environmental Impacts of the Universidad del Atlántico were used. The environmental impacts and their assessment were identified as a complement to the preparation of the ecobalance to measure ecoefficiency in the cooperatives. To determine the ecobalance, water consumption, electricity consumption, materials consumption, waste management and $\mathrm{CO} 2$ emissions were evaluated. In COOPAIN, three types of pollution were identified: the generation of non-hazardous waste with a low to medium impact, air emissions and noise pollution with medium impact levels, and the existence of energy and water consumption with a medium impact. As for the ecobalance, it obtained higher consumption of water and electricity. On the other hand, only one type of pollution was identified in CACD, the generation of non-hazardous waste and the existence of energy consumption, both at a low impact level. With regard to the ecobalance, it obtained greater consumption of materials, greater waste management and higher $\mathrm{CO} 2$ emissions. Finally, the eco-efficiency index indicated that CACD was relatively more eco-efficient than COOPAIN.
\end{abstract}

Keywords: eco-efficiency; water consumption; energy consumption; material consumption; waste management; $\mathrm{CO} 2$ emissions.

\footnotetext{
${ }^{1}$ Universidad Nacional Toribio Rodríguez de Mendoza de Amazonas, Facultad de Ingeniería Zootecnista, Agronegocios y Biotecnología, Chachapoyas, Perú

"Autor de Correspondencia, e-mail: reiner.reategui@untrm.edu.pe
} 


\section{INTRODUCCIÓN}

La industrialización económica conlleva la generación de avances en las tecnologías para hacer la vida del hombre más cómoda. Normalmente se centran en la producción más eficiente, en serie y a gran escala. Sin embargo, este crecimiento no se ha dado a la par con un cuidado o planeación sustentable de los recursos, que permitan la regeneración de los mismos en sus ciclos respectivos (Inda y Vargas, 2012). En Sudamérica, y en concreto en Perú, la actividad agrícola posee diversos factores que ocasionan la falta de rentabilidad y competitividad. Estos factores son los precios bajos y distorsionados, la débil articulación de la oferta con el mercado y la demanda urbana e industrial, los deficientes canales de comercialización, el mal funcionamiento de los mercados agrarios intangibles, o el escaso capital humano entre otros (POF, 2011). De los factores mencionados el más importante en Perú son los precios bajos y distorsionados ocasionados por los intermediarios, debido a que la rentabilidad de cualquier actividad económica es el factor que definirá la subsistencia de la actividad, y por tanto el desarrollo de la familia campesina. Por este motivo, se empezaron a crear cooperativas con el fin de combatir a los comerciantes intermediarios que pagan precios bajos por sus productos (Alonso-Rodrigo y Santa Cruz-Ayo, 2012). Las cooperativas agrarias suelen ser "empresas" con visión orgánica, ya que exigen a sus socios uno manejos orgánicos para sus fincas (Ren et al., 2018). Actualmente, existen demandas sociopolíticas que obligan a empresas a prestar mayor atención a su desempeño ambiental. La presión legal, el mercado y la opinión pública son los elementos con mayor peso, en la toma decisiones de carácter ambiental en la empresa. Para cumplir con estas obligaciones se aplica la ecoeficiencia, que es una estrategia para optimizar el uso de la energía, insumos y procesos de generación de bienes y servicios en general (Austermühle, 2012). Con ello, se busca aumentar la competitividad, además prevenir y minimizar los impactos ambientales.

Observando la importancia de la ecoficiencias dentro de las cooperativas peruanas, el objetivo de la investi- gación fue evaluar el índice de ecoeficiencia de dos cooperativas agrarias de la Región de Huánuco, la Cooperativa Agraria Industrial Naranjillo (COOPAIN) y la Cooperativa Agraria Cafetalera Divisoria (CACD). Al mismo tiempo se estableció cual tiene mejor índice, teniendo en cuenta que COOPAIN cuanta con mayor inversión, experiencia y tiempo en el mercado.

\section{MATERIAL Y MÉTODOS}

Elaboración del ecobalance

La evaluación de la ecoeficiencia se realizó en los meses de septiembre a diciembre en el año 2018. Para ello se usó la metodología del MINAN (2009), que indica que para determinar la ecoeficiencia se debe registrar al menos cinco indicadores, que reflejen la medición en el uso de los recursos naturales, así como el impacto en ellos, que contribuyen con el desarrollo de la actividad económica (Tabla 1). Esta medición es realizada a través del ecobalance permitiendo la comparación con otras empresas.

Tabla 1. Indicadores registrados para determinar la ecoeficiencia

\begin{tabular}{cc}
\hline Indicadores & Unidad de medida \\
\hline Consumo de agua & $\mathrm{m}^{3} /$ trabajador $/ \mathrm{mes}$ \\
Consumo de energía & $\mathrm{kWh} /$ trabajador $/ \mathrm{mes}$ \\
Consumo de materiales & $\mathrm{kg} /$ trabajador $/ \mathrm{mes}$ \\
Gestión de residuos & $\mathrm{kg} /$ trabajador $/ \mathrm{mes}$ \\
Emisiones $\mathrm{CO}_{2}$ & $\mathrm{~kg} /$ trabajador $/ \mathrm{mes}$ \\
\hline
\end{tabular}

Fuente: Basado en MINAM (2009)

El consumo de agua se obtuvo a través de las facturas de las empresas proveedoras del servicio, cuando es obtenida de la red pública. Pero como en ambas cooperativas también hay consumo de agua subterránea, se calculó un estimado del consumo, mediante el monitoreo de los medidores en los puntos de recolección de agua subterránea (MINAM, 2009)

La información sobre el consumo de energía se obtuvo a través de facturas de las empresas proveedoras del servicio, así como las facturas de compras de combustibles para la generación eléctrica con grupos electrógenos. La información sobre el consumo de materiales, en este caso sobre el consumo de papel, se obtuvo a través de las facturas de empresas proveedoras. Para determi- 
nar el peso de las hojas, se tomó en cuenta las características técnicas del papel bond. Por lo que una hoja de papel bond cuyo gramaje es $75 \mathrm{~g} / \mathrm{m}^{2}$, su peso será de 4,6875 g. Para la gestión de residuos, primero se realizó un reconocimiento en las oficinas donde se hace uso del papel bond. Se colocaron bandejas de plástico en cada oficina, donde cada uno de los trabajadores fueron colocados los papeles re-usados por los mencionados. El conteo de los papeles re-usados se hizo cada 3 días, donde se dejaba una pequeña " $X$ " en la hoja, con la finalidad de reconocerlo y no tomarlos en cuenta para el próximo conteo si se llegase a volver a colocar en la bandeja. La información sobre emisiones de $\mathrm{CO}_{2}$ se obtuvo a través de facturas de compras de combustibles, cuyo factor de conversión emisión de $\mathrm{CO}_{2}$ en $\mathrm{kg}$ es 8,87 $\mathrm{kg}$ de $\mathrm{CO}_{2}$ por galón de combustible (MINAN, 2009)

\section{Evaluación de la Ecoeficiencia}

Niveles máximos y mínimos

Una vez recolectados los datos de los indicadores con sus respectivas unidades de medida, se determinaron los valores máximos y mínimos de cada indicador en las dos cooperativas (Sepúlveda, 2008).

\section{Estandarización de indicadores}

Un indicador puede relacionarse de manera negativa o positiva con la ecoeficiencia, con respecto a lo que se considera una situación superior. De esta forma, si un aumento en el valor del indicador resulta en una mejoría del sistema, se considera que se tiene una relación positiva. Por el contrario, si un aumento en el valor del indicador empeora la situación, se tiene una relación inversa o negativa (Sepúlveda, 2008).

En el caso de las dos cooperativas en estudio, si la relación de consumo de agua, consumo de energía, consumo de materiales, y emisiones $\mathrm{CO}_{2}$, es inversamente proporcional con la ecoeficiencia, es decir, a mayor consumo menor ecoeficiencia, por tato la relación sería negativa, y se aplicó la ecuación (1).

$$
\mathrm{f}(\mathrm{x})=(\mathrm{x}-\mathrm{M}) /(\mathrm{m}-\mathrm{M})(1)
$$

Si la relación del consumo de gestión de residuos, depende de la cantidad de hojas reusadas, la relación será positiva (+). Por tanto, a mayor reúso de papel bond mayor ecoeficiencia, y se aplicó la ecuación(2).

$$
\mathrm{f}(\mathrm{x})=(\mathrm{x}-\mathrm{m}) /(\mathrm{M}-\mathrm{m})(2)
$$

Dónde:

$\mathrm{x}=$ Es el valor correspondiente del indicador para una unidad de análisis determinada en un período determinado.

$\mathrm{M}=$ Es el nivel máximo en periodo determinado

$\mathrm{m}=$ Es el valor mínimo de la variable en un período determinado.

Mediante la utilización de estas ecuaciones se obtuvieron los índices individuales para cada indicador, los cuales fluctúan entre 0 y 1 . Para ambos casos, cuando los indicadores representan una relación positiva o negativa, un valor de 1 representa la mejor situación, mientras que un valor 0 , representa la peor situación (Sepúlveda 2008). Por último, se utilizó el promedio, en el caso de aquellos indicadores que contienen subindicadores.

\section{Ponderación de indicadores}

Para obtener los pesos o ponderadores de los indicadores de ecoeficiencia, se usó el siguiente método Delphi (Astigarraga, 2003). Para ello, se elaboraron dos consultas o encuestas a expertos de ambas cooperativas y a expertos externos, con la finalidad de asignar una ponderación o puntaje de acuerdo al nivel de importancia en la ecoeficiencia, para cada indicador (Tabla 2).

Tabla 2. Valores de importancia para cada indicador

\begin{tabular}{cc}
\hline Evaluación & Puntaje \\
\hline Muy importante & 5 \\
Importante & 4 \\
Importancia regular & 3 \\
Poco importante & 2 \\
No importante & 1 \\
\hline
\end{tabular}

Fuente: Astigarraga (2003)

El lanzamiento de la segunda consulta, busco la convergencia de los expertos en base a disminuir la dispersión precisando la mediana (Landeta, 1999). Para obtener la ponderación de cada indicador, se sumó los valores obtenidos de cada indicador por cada 
respuesta de los expertos, se divide entre la suma total valores obtenidos de cada indicador conforme lo indica (Garmendia et al., 2005).

$$
P i=\frac{\sum \text { indicador } i}{\sum \text { total de los indicadores }}
$$

Donde:

Pi: Ponderación del indicador "i”".

\section{Índice de ecoeficiencia}

Luego de obtener los subíndices de todos los indicadores, estos se agregaron para obtener el índice integrado. La agregación, consistió en sumar los subíndices de los indicadores de ecoeficiencia para cada unidad de análisis.

$$
\text { Índice de ecoeficiencia }=\sum_{i=1}^{n} M_{i} * P_{i}
$$

Donde:

$\mathrm{M}_{\mathrm{i}}=$ Valor adimensional del indicador ( 0 a 1$)$. $\mathrm{P}_{\mathrm{i}}=$ Ponderación del indicador $(0 \mathrm{a} 1)$.

\section{Análisis estadístico}

Se usó la Prueba de hipótesis para la diferencia de medias poblacionales (PHDMP) o conocido también como prueba de $\mathrm{T}$ para muestras independientes, para la comparación de los indicadores y el índice de ecoeficiencia. Todos los análisis se realizaron con el software estadístico IBM SPSS Statistics Subscription Trial (IBMid: 7813080).

\section{RESULTADOS}

\section{Ecobalance de las cooperativas}

Los indicadores de consumo de agua y energía, gestión de residuos y emisión de $\mathrm{CO}_{2}$ fueron mayores en la cooperativa COOPAIN, mientras que el consumo de materiales fue mayor en la cooperativa CACD (Tabla 3 ).

Tabla 3. Ecobalance de las cooperativas en estudio.

\begin{tabular}{ccc}
\hline Indicadores & CACD & COOPAIN \\
\hline Consumo de agua & 5,835 & 33,089 \\
Consumo de energía & 128,355 & 621,422 \\
Consumo de materiales & 0,455 & 0,07 \\
Gestión de residuos & 0,066 & 0,028 \\
Emisión de $\mathrm{CO}_{2}$ & 33,613 & 23,37 \\
\hline
\end{tabular}

\section{Determinación del índice de ecoeficiencia.}

En la Tabla 4 se observa que el indicador de consumo de agua (ICA), el indicador de consumo de energía (ICA) y el indicador de gestión de residuos (IGR) de la cooperativa CACD son mayores que los de la COOPAIN. Por otro lado, el indicador de consumo de materiales (ICM) y el indicador de emisión de $\mathrm{CO}_{2}$ (IEDC), fueron mayores en la cooperativa COOPAIN.

\begin{tabular}{|c|c|c|c|c|}
\hline Cooperativas & Indicadores & $\begin{array}{c}\text { Datos } \\
\text { estandarizados (DA) }\end{array}$ & Ponderación (P) & $\begin{array}{c}\text { Factorizando (DA } \\
\text { x P) }\end{array}$ \\
\hline \multirow{6}{*}{ CACD } & ICA & 0,968 & 0,208 & 0,201 \\
\hline & ICE & 0,988 & 0,204 & 0,202 \\
\hline & ICM & 0,282 & 0,192 & 0,054 \\
\hline & IGR & 0,575 & 0,208 & 0,120 \\
\hline & IEDC & 0,256 & 0,188 & 0,048 \\
\hline & & ice de ecoeficiencia & & 0,625 \\
\hline \multirow{6}{*}{ COOPAIN } & ICA & 0,011 & 0,208 & 0,002 \\
\hline & ICE & 0,306 & 0,204 & 0,062 \\
\hline & ICM & 0,953 & 0,192 & 0,183 \\
\hline & IGR & 0,135 & 0,208 & 0,028 \\
\hline & IEDC & 0,819 & 0,188 & 0,154 \\
\hline & & ice de ecoeficiencia & & 0,430 \\
\hline
\end{tabular}

Tabla 4. Índices de ecoeficiencia de las cooperativas en estudio.

La prueba de hipótesis para la diferencia de medias poblacionales practicada a los indicadores y al índice de ecoeficiencia, indica que solo existe igualdad estadística en el IGR (Tabla 5).

\section{DISCUSIÓN}

Para el indicador del consumo de agua, el valor es más alto en la cooperativa COOPAIN que en la CACD, siendo estos mayores a la dotación mínimas estableci- 
Tabla 5. Evaluación estadística de los indicadores y del índice de ecoeficiencia de las cooperativas en estudio

\begin{tabular}{ccc}
\hline Indicadores & CACD & COOPAIN \\
\hline ICA & $0,201 \mathrm{~A}$ & $0,002 \mathrm{~B}$ \\
ICE & $0,202 \mathrm{~A}$ & $0,062 \mathrm{~B}$ \\
ICM & $0,054 \mathrm{~A}$ & $0,183 \mathrm{~B}$ \\
IGR & $0,120 \mathrm{~A}$ & $0,028 \mathrm{~A}$ \\
IEDC & $0,048 \mathrm{~A}$ & $0,154 \mathrm{~B}$ \\
\hline Indice de & $0,625 \mathrm{~A}$ & $0,430 \mathrm{~B}$ \\
ecoeficiencia & & \\
\hline
\end{tabular}

Letras diferentes en una misma fila indica significancia estadística $(\mathrm{p}<0,05)$

das por el MVCS (2011), que indica que el agua para consumo industrial deberá calcularse de acuerdo a la naturaleza de la industria y su proceso de manufactura. En los locales industriales la dotación de agua para consumo humano en cualquier tipo de industria, será de 2,4 $\mathrm{m}^{3}$ por trabajador al mes. Por su parte, MINAM (2015) realizó una evaluación de ecoeficiencia en su Sede Central, encontrando un consumo de agua de $6,263 \mathrm{~m}^{3} /$ persona/mes. Mientras que OSINERGMIN (2013), realizó una evaluación similar encontrando en su sede central un consumo de agua de 0,895 $\mathrm{m}^{3} /$ persona/mes. El consumo de energía en ambas cooperativas es considerablemente alto para el consumo promedio mensual del Perú en el 2012, que fue 106 kWh/persona/mes (OSINERGMIN, 2015).

En cuanto al consumo de materiales, es bajo al compararlo con el consumo de papel de 6,7417 $\mathrm{kg} /$ persona/mes encontrados en un estudio realizado por MINAM (2015) en su sede central. Mientras que OSINERGMIN (2013), realizó una evaluación similar encontrando en su sede central para el consumo de papel fue de 0,6719 kg/persona/mes. Según el MINAM (2009), para producir una tonelada de papel se necesitan 3 árboles medianos o 2385 kilogramos de madera, 440000 litros de agua dulce y limpia y $7600 \mathrm{kWh}$ de energía eléctrica. Según lo mencionado por cada trabajador de la CACD por año estarían gastando $13,022 \mathrm{~kg}$ de madera, 2402,4 litros de agua dulce y 41,496 kWh de energía eléctrica. Mientras que la COOPAIN estaría gastado por trabajador al año $2 \mathrm{~kg}$ de madera, 369,6 litros de agua dulce y $6,384 \mathrm{kWh}$ de energía eléctrica. En lo referente a la gestión de residuos, mensualmente por trabajador la CACD estaría evitando la emisión de
59,4 g de $\mathrm{CO}_{2}$ y la COOPAIN 25,2 $\mathrm{g}$ de $\mathrm{CO}_{2}$. Al reducir el uso de papel se evita la generación de residuos, el consumo de los recursos naturales (madera, agua y energía) y los problemas de contaminación que lleva aparejada la producción de papel (MINAM, 2009).

La emisión de $\mathrm{CO}_{2}$ fue más alta en la CACD que en la COOPAIN. Sin embargo, estos datos son bajos si se comparan con el promedio peruano, el cual esta establecido en la emision de 4,7 toneladas de $\mathrm{CO}_{2}$ por persona al año, siendo una cifra menor a otros países de la región (Gutiérrez, 2015). Por su parte Álvarez (2008), determino que una persona genera aproximadamente $1,14 \mathrm{~g}$ de $\mathrm{CO}_{2} /$ día, por lo que en el mundo entero se genera diariamente 2500 millones de toneladas de $\mathrm{CO}_{2}$, los cuales es mitigada naturalmente por la fotosíntesis.

Los índices de ecoeficiencia de ambas cooperativas son estadísticamente diferentes (Tabla 4 y 5). Esta diferencia se debe principalmente a factores. La cooperativa COOPAIN realiza mayores procesos industriales que requieren de agua, algunos de estos procesos son obtención de licor de cacao y manteca de cacao, elaboración de chocolate de taza y torta de cacao, así como la producción de cacao en polvo. Como indica Pascual (2018), al igual que en el resto de la industria láctea, el agua residual generada en este sector procede de las operaciones de lavado, así como de los equipos de esterilización y enfriamiento.

Al mismo tiempo, la cooperativa COOPAIN realiza mayores tipos de operaciones al tener un ciclo productivo más complejo. Como indica Melo (2015) la mitigación de los impactos ambientales es más sencillo en nuevas empresas, indicando también, que al pensar en impacto ambiental de las empresas se tiene que partir de dos premisas: 1) todas las empresas tienen impactos ambientales en su operación y tienen la responsabilidad de hacerse cargo por ellos, y 2) no todos los impactos son iguales, pues dependen del tipo de operaciones de producción que tenga la empresa, del tipo de decisiones que se tomen al respecto de ésta, y por supuesto, del sector. Por otro lado, la CACD posee en promedio 19 trabajadores y la COOPAIN 83 trabajadores, por lo que 
empresarialmente la COOPAIN es más grande. Las pequeñas empresas tienen un personal de entre 11 a 49 trabajadores, mientras que las medianas empresas tienen un personal de entre 50 a 250 trabajadores (Nicuesa, 2016). Por lo general, las empresas que tienen mayores inversiones tienden a realizar mayor impacto ambiental. En el mundo existen muchas empresas con diferentes giros industriales, y la gran mayoría de ellas tienen un impacto ambiental principalmente en el aire, agua y suelo, al igual que en la flora y fauna (Carranza, 2015). Cabe destacar, que todas las organizaciones están generando impactos en el medio ambiente a lo largo de todo su proceso productivo, desde la extracción de materias primas, pasando por el consumo de energía y la generación de residuos y emisiones, tanto por lo que a ellas mismas se refiere como por parte de los consumidores que adquieren sus productos (Barco, 2013).

La cooperativa CACD con un índice de ecoeficiencia de 0,625 , indica que se encuentra en un sistema estable. Mientras que la cooperativa COOPAIN posee un índice de ecoeficiencia de 0,43 , que corresponde a un sistema inestable. Si se aplicara un sistema de cinco colores o biograma, un índice por debajo de 0,2 se representa en color rojo, simbolizando un estado del sistema con una alta probabilidad de colapso. Para los niveles entre 0,2 y 0,4 se usa el color anaranjado, indicando una situación crítica. De 0,4 a 0,6 se usa el color amarillo, correspondiendo a un sistema inestable. De 0,6 a 0,8 la representación es en azul, simbolizando un sistema estable. Finalmente, de 0,8 a 1 el color es verde y se considera como la situación óptima del sistema. (Sepúlveda, 2008).

\section{CONCLUSIONES}

La CACD y la COOPAIN consumen por trabajador por mes 5,835 y $33,089 \mathrm{~m}^{3}$ de agua; $128,355 \mathrm{kWh}$ y $621,422 \mathrm{kWh}$ de energía eléctrica; 0,455 kg y 0,07 kg de papel bond; reusando $0,066 \mathrm{~kg}$ y $0,028 \mathrm{~kg}$ de papel bond y emitiendo $33,613 \mathrm{~kg}$ y $23,37 \mathrm{~kg}$ de $\mathrm{CO}_{2}$, respectivamente para todos los casos. La CACD posee un nivel de producción bajo al lado de la COOPAIN, siendo esto inversamente proporcional al índice de ecoeficiencia de cada empresa.

\section{REFERENCIAS BIBLIOGRÁFICAS}

Alonso-Rodrigo, E. y I. Santa Cruz-Ayo. 2012. "Identidad, competitividad y creación de empleo: retos para una nueva fiscalidad de las cooperativas de trabajo asociado." Boletín de la Asociación Internacional de Derecho Cooperativo (50): 73-11. DOI: 10.18543/baidc-502016pp73-101.

Álvarez, J. 2008. ¿Cuánto CO2 emitimos los humanos al respirar? https://foro.tiempo. com/relacion-co2 -natural-humanorespiracion-t104553.0.html (Consultada el 15 de marzo de 2017)

Astigarraga, E. 2003. El Método Delphi. Bilbao (España): Universidad de Deusto.

Austermühle, S. 2012. Sostenibilidad y ecoeficiencia en la empresa moderna. Lima (Perú): Universidad Peruana de Ciencias Aplicadas.

Barco, J. M. 2013. Impacto medioambiental de la actividad empresarial. http://blogsegurida dindustrial.com/el-impacto-ambiental-delas-empresas/ (Consultada el 26 de marzo de 2018)

Carranza, L. 2015. EL impacto ambiental de las empresas. http://www.responsabilidadso cialempresarial.com/?p=260 (Consultada el 26 de marzo de 2018)

Garmendia, A., A, Salvador, C. Crespo, y L. Garmendia. 2005. Evaluación de impacto ambiental. Madrid(España): Pearson Prentice Hall.

Gutiérrez, S. 2015. Día del medio ambiente. http://larepublica.pe/sociedad/5523-pacificoseguros-un-peruano-promedio-emite-47toneladas-de-co2-al-ano (Consultada el 31 de marzo de 2018)

Inda, C. M. y J. G. Vargas. 2012. "Ecoeficiencia y competitividad: tendencias y estrategias con metas comunes." Revista de Ingeniería de Recursos Naturales y del Ambiente (11): 33- 
40.

Landeta, J. 1999. El método de Delphi. Barcelona (España): Ariel.

Melo, J. M. 2015. Impacto ambiental de la empresa: Volviendo a lo básico. http://labuenaempresa.com/2015/02/27/impa cto-ambiental-de-la-empresa/ (Consultada el 26 de marzo de 2018)

MINAM (Ministerio del Ambiente). 2009. Guía de Ecoeficiencia para Empresas. Lima (Perú): MINAM.

MINAM (Ministerio del Ambiente). 2015. "R.S.G. N ${ }^{\circ}$ 019-2015-MINAM. Plan de ecoeficiencia institucional Ministerio de Ambiente." Lima (Perú).

MVCS (Ministerio de Vivienda, Construcción y Saneamiento). 2011. Capítulo III: Normalización de infraestructura urbana y propuesta de estándares. Lima (Perú): MVCS.

Nicuesa, M. 2016. Cuatro tipos de empresa según su tamaño. https://empresariados.com/cuatrotipos-de-empresa-segun-su-tamano (Consultada el 02 de abril de 2018)

Pascual, N. 2018. Depuración de aguas residuales industriales: elaboración de cacao y chocola$t$ $e$ $s$

http://depuradorasaguasresiduales.es/depura cion-de-aguas-residuales-industriales-en-laindustria-del-chocolate/ (Consultada el 02 de abril de 2018)

OSINERGMIN (Organismo Supervisor de la Inversión en Energía y Minería). 2013. Diagnóstico de ecoeficiencia. Informe Técnico. Lima (Perú).

OSINERGMIN (Organismo Supervisor de la Inversión en Energía y Minería). 2015. Encuesta Residencial de Uso y Consumo de Energía. ERCUE 2014-2015. Informe Resultados. Lima (Perú).

POF (Peru Opportunity Fund). 2011. Diagnóstico de la Agricultura en el Perú. Informe Final. Lima (Perú).
Ren, S., E. Li, Q. Deng, H. He, y S. Li. 2018. “Analysis of the impact of rural households' behaviors on heavy metal pollution of arable soil: Taking Lankao County as an example." Sustainability 10 (12): 4368. DOI: 10.3390/su10124368.

Sepúlveda, S. 2008. Metodología para estimar el nivel de desarrollos sostenible de territorios. San José (Costa Rica): Instituto Interamericano de Cooperación para la Agricultura. 